\title{
Studying the infrared region in Landau gauge QCD*
}

\section{A. Sternbeck ${ }^{\dagger}$}

Humboldt-Universität zu Berlin, Institut für Physik, D-12489 Berlin, Germany

E-mail: andre.sternbeck@physik.hu-berlin.de

\section{E.-M. Ilgenfritz}

Humboldt-Universität zu Berlin, Institut für Physik, D-12489 Berlin, Germany

E-mail: ilgenfri@physik.hu-berlin.de

\section{Müller-Preussker}

Humboldt-Universität zu Berlin, Institut für Physik, D-12489 Berlin, Germany

E-mail: mmp@physik.hu-berlin.de

\section{A. Schiller}

Universität Leipzig, Institut für Theoretische Physik, D-04109 Leipzig, Germany

E-mail: Arwed.Schiller@itp.uni-leipzig.de

\begin{abstract}
We report on the progress we made in studying the infrared behavior of the ghost and gluon dressing functions in Landau gauge. Related to this we also investigate a running coupling given in terms of those functions and compare our results to those coming from the Dyson-Schwinger approach. We present first numerical results for the $S U(3)$ ghost-ghost-gluon vertex renormalization constant. In addition the spectrum of low-lying eigenvalues and eigenfunctions of the Faddeev-Popov operator is determined. The saturation of the ghost propagator in terms of those eigenvalues and eigenmodes is discussed at lower momenta.
\end{abstract}

XXIIIrd International Symposium on Lattice Field Theory

25-30 July 2005

Trinity College, Dublin, Ireland

*This work has been supported by the DFG under contract FOR 465.

${ }^{\dagger}$ Speaker. Supported by the DFG-funded graduate school GK 271. 


\section{Introduction}

The predicted infrared behavior of both the gluon and ghost propagator in QCD was the starting point of our combined investigation of those Green's functions. In fact, solutions of a truncated set of Dyson-Schwinger equations (DSE) for both propagators in Landau gauge predict their dressing functions $Z_{D}$ and $Z_{G}$ to follow the power laws [1,2,3]

$$
Z_{D}\left(q^{2}\right) \propto\left(q^{2}\right)^{2 \kappa} \quad \text { and } \quad Z_{G}\left(q^{2}\right) \propto\left(q^{2}\right)^{-\kappa} \quad \text { with } \quad \kappa \approx 0.595
$$

at (extremely) low momentum. Thus, an infrared vanishing gluon propagator occurs in intimate connection with a diverging ghost propagator. The latter would be in agreement with the Zwanziger-Gribov horizon condition as well as with the Kugo-Ojima confinement criterion $[4,5]$.

Furthermore, in terms of the gluon and ghost dressing functions a running coupling $\alpha_{s}\left(q^{2}\right)$ with a momentum dependence

$$
\alpha_{S}\left(q^{2}\right)=\frac{g_{0}^{2}}{4 \pi} Z_{G}^{2}\left(q^{2}\right) Z_{D}\left(q^{2}\right) \quad \text { with } g_{0}^{2}=6 / \beta \text { for } S U(3)
$$

can be defined. This construction is based on the consideration of the ghost-ghost-gluon vertex where the renormalization function $Z_{1}$ of this vertex is assumed to be constant at all momenta. Given the asymptotic behavior in Eq. (1.1) the running coupling would have a finite limit at zero momentum [2].

Those infrared properties are claimed to be independent of how the DSEs are truncated. However, it is worthwile to cross-check them using lattice simulations [6]. We have been pursuing this for the $S U(3)$ case during the last two years [7]. In addition, we have begun to study the ghostgluon vertex and the spectral properties of the Faddeev-Popov operator. The low-lying eigenmodes of the latter are believed to be immediately reflected by a diverging ghost propagator.

\section{The dressing functions and the running coupling}

We have simultaneously studied the gluon and ghost propagators in the quenched approximation. Thermalized $S U(3)$ gauge field configurations $U=\left\{U_{x, \mu}\right\}$ have been put into the Landau gauge. It is well-known that the Landau gauge functional

$$
F_{U}[g]=\frac{1}{4 V} \sum_{x} \sum_{\mu=1}^{4} \mathfrak{R e} \operatorname{Tr}^{g} U_{x, \mu} \quad \text { with } \quad g_{x} \in S U(3)
$$

has several local maxima (Gribov copies), each satisfying the lattice Landau gauge condition $\partial_{\mu} A_{\mu}=0$. To explore to what extent this ambiguity has a significant influence on gauge dependent observables, we have gauge-fixed each thermalized configuration $N_{\mathrm{cp}}$ times using the overrelaxation algorithm, starting from a random gauge copy. For each configuration $U$, we have selected the first $\left(f_{c}\right)$ and the best $(\mathrm{bc})$ gauge copy (that with the largest functional value) for subsequent measurements. For details we refer to our recent publication [7].

It turns out that the Gribov ambiguity has no systematic influence on the infrared behavior of the gluon propagator. In marked contrast to this, the ghost propagator at lower momenta depends on the selection of gauge copies. This can be seen in Fig. 1 where in the upper panel the ghost 
dressing function $Z_{G}$ is presented as a function of momentum $q$. The lower panel shows the ratio of the corresponding functions measured on $\mathrm{f}_{\mathrm{c}}$ and $\mathrm{bc}$ gauge copies. We observe that the influence of the Gribov ambiguity becomes important at momenta $q<1 \mathrm{GeV}$. However, the ratio for $\beta=6.0$ is larger than the corresponding ratio for $\beta=5.8$ in the sensitive momentum region at the same lattice size $24^{4}$. Therefore, we conclude that the systematic Gribov copy effect at the same momentum is reduced if the physical volume is increased.

We have first studied the infrared behavior of the gluon and ghost dressing functions. In particular, we have tried to confirm the proposed power laws Eq. (1.1). However, fitting both functions for $q<0.5 \mathrm{GeV}$ we were unable to extract a common exponent $\kappa>0.5 .^{1}$ This situation is also reflected in the infrared behavior of the running coupling $\alpha_{S}\left(q^{2}\right)$ which is proportional to the product $Z_{G}^{2}\left(q^{2}\right) Z_{D}\left(q^{2}\right)$ of the two dressing functions (cf. the left hand side of Fig. 2). There the fits to the 1-loop and 2-loop running coupling are also shown.

One clearly sees that the running coupling monotonously increases with decreasing momentum as long as $q^{2}>0.4 \mathrm{GeV}^{2}$. After passing a maximum $\alpha_{s}\left(q^{2}\right)$ decreases. One could blame finite volume effects for this disagreement with the continuum predictions of the DSE approach.

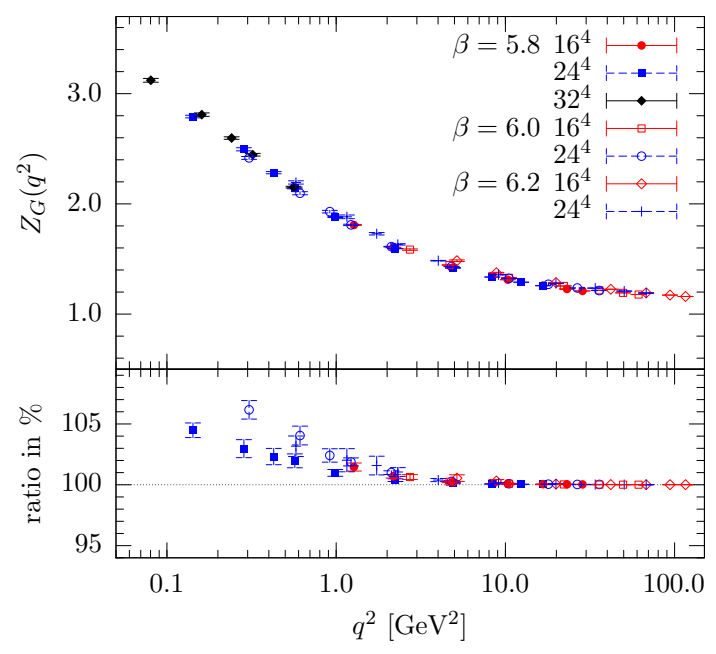

Figure 1: The ghost dressing function $Z_{G}$ measured on bc gauge copies is shown vs. the (physical) momentum $q^{2}$. The lower panel shows the ratio $\left\langle Z^{\mathrm{fc}}\right\rangle /\left\langle Z^{\mathrm{bc}}\right\rangle$ of dressing functions determined on first $\left(f_{C}\right)$ and best $(\mathrm{bc})$ gauge copies.

Indeed, recent DSE investigations [8] on a torus also show a modified infrared behavior of the gluon and ghost dressing functions. One could also question whether the ghost-ghost-gluon vertex renormalization function $Z_{1}\left(q^{2}\right)$ is really constant at lower momenta. A recent investigation dedicated to the ghost-ghost-gluon-vertex renormalization function $Z_{1}\left(q^{2}\right)$ for the case of $S U(2)$ gluodynamics [9] supports that $Z_{1}\left(q^{2}\right) \approx 1$ at least for momenta larger than $1 \mathrm{GeV}$. We have performed a similar
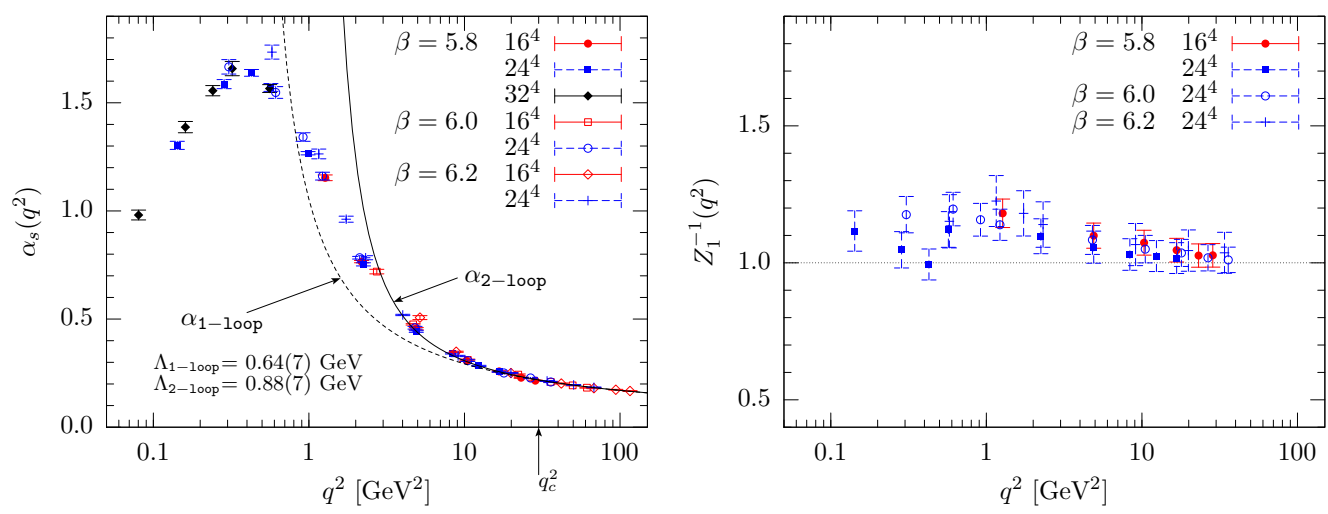

Figure 2: The momentum dependence of the running coupling $\alpha_{s}\left(q^{2}\right)$ (left) and of the ghost-ghost-gluon vertex renormalization function $Z_{1}\left(q^{2}\right)$ is shown. Both have been measured on bc gauge copies.

\footnotetext{
${ }^{1}$ We should mention that the number of data points in this momentum region is certainly too small to fit the asymptotic behavior for $q^{2} \rightarrow 0$
} 
study of $Z_{1}\left(q^{2}\right)$ for the case of $S U(3)$. Our (preliminary) results are presented on the right hand side of Fig. 2. So far the data do not allow to draw a final conclusion whether $Z_{1}\left(q^{2}\right)$ stays constant at lower momenta.

\section{Infrared properties of the Faddeev-Popov operator}

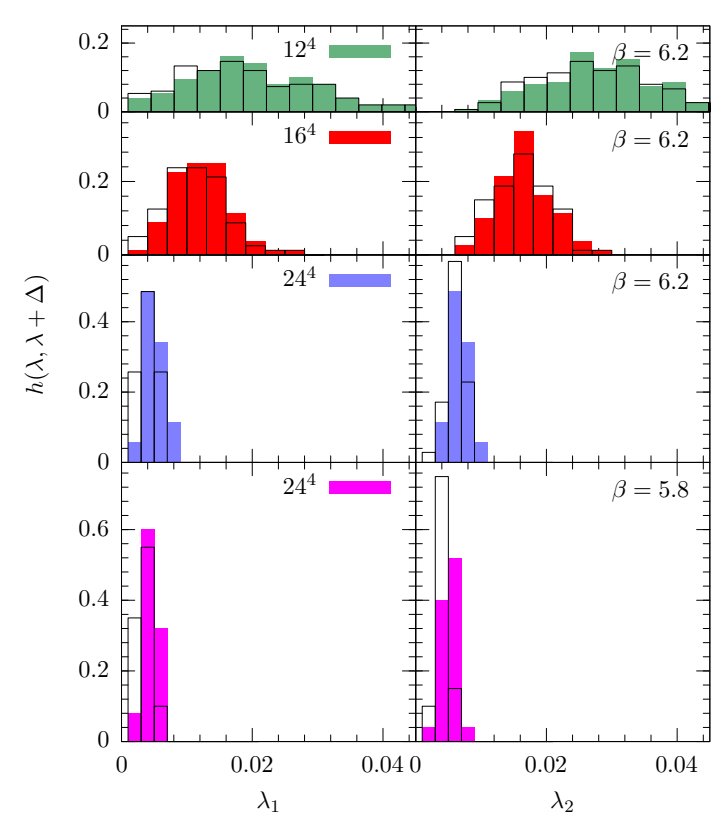

Figure 3: The distribution $h(\lambda)$ of the lowest (left panels) and second lowest (right panels) FaddeevPopov eigenvalues $\lambda_{1}$ and $\lambda_{2}$. Filled columns refer to bc gauge copies, while open columns refer to $\mathrm{fc}_{\mathrm{C}}$ copies.

the ghost propagator to diverge stronger than $1 / q^{2}$ near zero momentum [4].

At $\beta=6.2$ we have extracted the 200 (50) lowest (non-trival) eigenvalues and their corresponding eigenfunctions on a $12^{4}$ and $16^{4}\left(24^{4}\right)$ lattice. Additionally, 90 eigenvalues have been extracted on a $24^{4}$ lattice at $\beta=5.8$. This allows us to check that low-lying eigenvalues are shifted towards $\lambda=0$ as the physical volume is increased. In order to clarify how the choice of gauge copy influences the spectrum, the eigenvalues and eigenvectors have been extracted separately on our sets of $\mathrm{fc}$ and $\mathrm{bc}$ gauge-fixed configurations.

In Fig. 3 the distributions of the lowest $\lambda_{1}$ and second lowest $\lambda_{2}$ eigenvalues of the F-P operator are shown for different (physical) volumes. There $h(\lambda, \lambda+\Delta)$ represents the average number (per configuration) of eigenvalues found in the intervall $[\lambda, \lambda+\Delta]$. Open bars refer to the distribution on $\mathrm{fc}_{\mathrm{C}}$ gauge copies while full bars to that on bc copies. From this figure it is quite obvious that both eigenvalues, $\lambda_{1}$ and $\lambda_{2}$, are shifted to lower values as the physical volume is increased. In conjunction the spread of $\lambda$ values is decreased. We have found that the center of those distributions tends towards zero stronger than $1 / L^{2}$. Here $L$ refers to the linear lattice extension in physical 
units. For example, we have found $\left\langle\lambda_{1}\right\rangle(L) \propto 1 / L^{2+\varepsilon}$ with $\varepsilon \approx 0.16(4)$. It is also visible that the eigenvalues $\lambda_{1}$ and $\lambda_{2}$ on $f_{C}$ gauge copies are on average lower than those obtained on bc copies.

Eq. (3.1) suggests the popular belief that low-lying eigenvalues and eigenvectors have a major impact on the ghost propagator at the lowest momenta. Actually, this is not so easy to predict. We have studied, to what extent the lowest $n$ modes saturate the estimator of the ghost propagator $G(k)$ on a given set of gauge copies. We show the result in Fig. 4 for the lowest $\left(q_{1}\right)$ and the second lowest momentum $\left(q_{2}\right)$ available on lattices of different size at $\beta=6.2$. The estimates for $G_{n}(k)$ have been divided by the full (conjugate gradient) estimator $G(k)$ in order to compare the approach to saturation for different volumes.

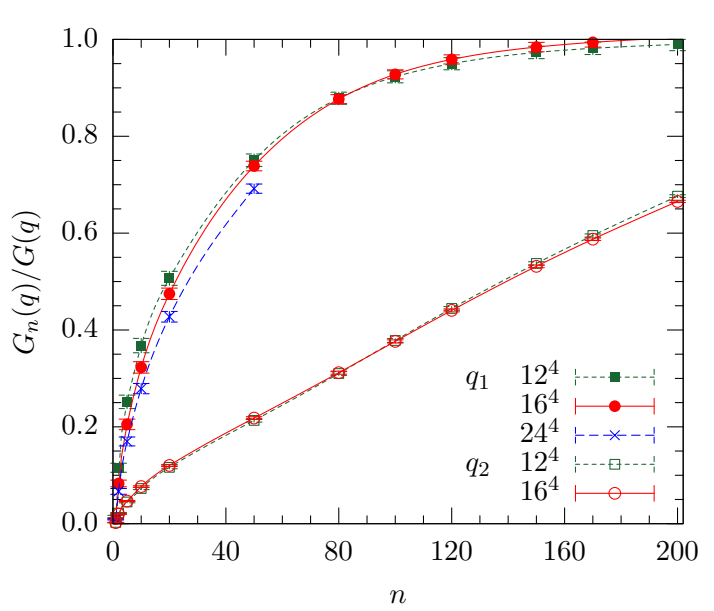

Figure 4: The ratio of the ghost propagator $G_{n}(q)$, approximated by the $n$ lowest F-P eigenmodes and eigenvalues, to the corresponding full estimator $G(q)$ is shown as a function of $n$ for the lowest $\left(q_{1}\right)$ and second lowest momentum $\left(q_{2}\right)$. The inverse coupling is $\beta=6.2$ and the lattice size ranges from $12^{4}$ to $24^{4}$.

there are, albeit few, relatively localized ones.

Considering first the lowest momentum $q_{1}$ we observe from Fig. 4 that the rates of convergence differ, albeit slightly, for the three different lattice sizes. The relative deficit rises with the lattice volume. For the $12^{4}$ and $16^{4}$ lattice the rates of convergence are still about the same. For example, taking only 20 eigenmodes is definitely too less. One is short of reproducing the ghost propagator (by about 50\%) whereas $150 \ldots 200$ eigenmodes are sufficient to reproduce it within a few percent. For the second lowest momentum $q_{2}$ even 200 eigenmodes are far from saturating the result.

In addition, the inverse participation ratio has been calculated for the modes in order to figure out the localization properties of the F-P eigenmodes. It turns out that the lowest eigenmodes are not localized on average, although

\section{Conclusions}

We have studied the lower momentum region of Landau gauge gluodynamics using Monte Carlo simulations on lattice sizes $16^{4}, 24^{4}$ and $32^{4}$. The inverse bare coupling constant has been set to $\beta=5.8,6.0$ and 6.2 using the Wilson action.

Concerning the influence of Gribov copies, it turns out that the gluon propagator does not depend on the chosen gauge copy while the ghost propagator does. There are indications, however, that the strength of this influence, at the same physical momentum, decreases with increasing volume. Towards $q \rightarrow 0$ in the infrared momentum region, the gluon dressing function is decreasing, while the ghost dressing function is increasing. However, the power laws predicted from the DSE approach cannot be confirmed from our data. Correspondingly, the behavior of the running coupling $\alpha_{s}\left(q^{2}\right)$ in a suitable momentum subtraction scheme (based on the ghost-ghost-gluon vertex) does not approach the expected finite limit. Instead, this coupling has been found to decrease for lower momenta after passing a turnover at $q^{2} \approx 0.4 \mathrm{GeV}^{2}$. 
In addition, we have investigated the spectral properties of the F-P operator and its relation to the ghost propagator on a $24^{4}$ lattice at $\beta=5.8$ and on $12^{4}, 16^{4}$ and $24^{4}$ lattices at $\beta=6.2$. As expected from another study [4] we have found that the low-lying eigenvalues are shifted towards zero (the configurations approaching the Gribov horizon) as the volume is increased. The lowlying eigenvalues extracted on bc gauge copies (those with the largest functional value) are larger on average than those on $f_{C}$ copies. Thus, for finite volumes better gauge-fixing (in terms of the gauge functional) keeps the configurations at larger distance from the Gribov horizon. The study of the contributions to the ghost propagator coming from the lowest eigenmodes of the F-P operator reveals that even the ghost propagator at the lowest momentum needs not less than $150 \ldots 200$ modes for being sufficiently approximated. This result refers to our smallest $\left(12^{4}\right)$ lattice at $\beta=6.2$. For larger volumes and higher lattice momenta the amount of modes needed in an eigenmode expansion is even larger.

All simulations have been done on the IBM pSeries 690 at HLRN. We thank Christian S. Fischer, Lorenz von Smekal and Anthony G. Williams for inspiring discussions. We are grateful to Hinnerk Stüben for contributing parts of the program code.

\section{References}

[1] L. von Smekal, R. Alkofer, and A. Hauck, Phys. Rev. Lett. 79 (1997) 3591 [hep-ph/9705242]; L. von Smekal, R. Alkofer, and A. Hauck, Ann. Phys. 267 (1998) 1 [hep-ph/9707327];

R. Alkofer and L. von Smekal, Phys. Rept. 353 (2001) 281 [hep-ph / 0007355$].$

[2] C. Lerche and L. von Smekal, Phys. Rev. D65 (2002) 125006 [hep-ph/ 0202194$].$

[3] D. Zwanziger, Phys. Rev. D65 (2002) 094039 [hep-th/ 0109224 ].

[4] D. Zwanziger, Phys. Rev. D69 (2004) 016002 [hep-ph/ 0303028$].$

[5] D. Zwanziger, Nucl. Phys. B412 (1994) 657;

V. N. Gribov, Nucl. Phys. B139 (1978) 1;

T. Kugo and I. Ojima, Prog. Theor. Phys. Suppl. 66 (1979) 1.

[6] J. C. R. Bloch, A. Cucchieri, K. Langfeld, and T. Mendes, Nucl. Phys. Proc. Suppl. 119 (2003) 736 [hep-lat/0209040].

[7] A. Sternbeck, E.-M. Ilgenfritz, M. Müller-Preussker, and A. Schiller, Phys. Rev. D72 (2005) 014507 [hep-lat/0506007].

[8] C. S. Fischer, B. Grüter, and R. Alkofer, hep-ph/0506053;

C. S. Fischer, R. Alkofer, and H. Reinhardt, Phys. Rev. D65 (2002) 094008 [hep-ph/ 0202195 ];

C. S. Fischer and R. Alkofer, Phys. Lett. B536 (2002) 177 [hep-ph / 0202202$]$;

C. S. Fischer, PoS(LAT2005) 330 these proceedings [hep-lat/0509031].

[9] A. Cucchieri, T. Mendes, and A. Mihara, JHEP 12 (2004) 012 [hep-lat / 0408034 ].

[10] J. Greensite, S. Olejnik, and D. Zwanziger, JHEP 05 (2005) 070 [hep-lat / 0407032 ].

[11] J. Gattnar, K. Langfeld, and H. Reinhardt, Phys. Rev. Lett. 93 (2004) 061601 [hep-lat/ 0403011 ]. 